\title{
Promoción de valores y políticas organizacionales a partir del derecho público en instituciones de seguridad ciudadana
}

\section{Promotion of organizational values and policies based on public law in citizen security institutions}

\author{
Edison Aroldo Gracia Panta \\ edigrapa@hotmail.com \\ Universidad Laica Eloy Alfaro de Manabí \\ Ecuador \\ https://orcid.org/0000-0001-8271-9139 \\ José Tranquilino Bermeo Reyes \\ josephber@gmail.com \\ Universidad Laica Eloy Alfaro de Manabí \\ Ecuador \\ https://orcid.org/0000-0002-7847-5378 \\ Liliana Hipatía Rodríguez Mera \\ hipati710321@yahoo.com \\ Universidad Laica Eloy Alfaro de Manabí \\ Ecuador \\ https://orcid.org/0000-0003-4087-4362
}

Recibido: 24 de abril de 2019

Aprobado: 30 de mayo de 2019

\section{RESUMEN}

El objetivo de la investigación fue determinar los efectos de un programa de formación en promoción de valores y políticas organizacionales a partir del derecho público en instituciones de seguridad ciudadana. Tipo de investigación obedece al explicativo, con un diseño cuasi experimental de pre prueba, postprueba y grupo control. En la postprueba el nivel de significancia bilateral cambió a "0", lo que es lo mismo < 0,05, lo que indica diferencias significativas en ambos grupos (experimental y control). Se rechaza $\mathrm{HO}$ y se acepta $\mathrm{H} 1$, lo que indica que el tratamiento aplicado resultó favorable a la muestra objeto de estudio, con lo cual se considera que la ciudadanía cuenta con servidores en posibilidad de abordar desde el derecho público, una asertiva relación interpersonal basada en valores para el buen vivir. 
Descriptores: Derecho público; Sistema jurídicos; Deontología; Seguridad del Estado.

\begin{abstract}
The objective of the research was to determine the effects of a training program in promoting values and organizational policies based on public law in citizen security institutions. Type of research is due to the explanatory, with a quasi-experimental design of pre-test, post-test and control group. In the post-test the level of bilateral significance changed to "0", which is the same $<0.05$, which indicates significant differences in both groups (experimental and control). $\mathrm{HO}$ is rejected and $\mathrm{H} 1$ is accepted, which indicates that the treatment applied was favorable to the sample under study, which means that citizens have servers capable of addressing, from public law, an assertive interpersonal relationship based on values for good living.
\end{abstract}

Descriptors: Public law; Legal systems; Deontology; State security

\title{
INTRODUCCIÓN
}

Los valores y políticas organizacionales juegan un papel fundamental para establecer organizaciones con una visión deontológica que posibilite a sus integrantes a trabajar bajo una concepción bioética de interrelación con sus semejantes, sea dentro o fuera de la institución, articulándose un comportamiento equilibrado para promover acciones en favor del fortalecimiento de los derechos humanos como factor elemental para la convivencia y seguridad ciudadana.

Así desde el enfoque del derecho público, se promovió la formación de servidores públicos pertenecientes a instituciones de seguridad ciudadana de la ciudad de Manta, Ecuador, con la finalidad de promover acciones contribuyentes a la consolidación de interrelaciones personales que permitan la promoción de conductas favorables al buen vivir, por lo tanto, se procedió a aplicar un programa de formación en promoción de valores y políticas organizacionales a partir del derecho público en instituciones de seguridad ciudadana, procurándose determinar su efectividad, para lo cual se aplicó a un grupo control y experimental, por lo tanto, el objetivo de la investigación fue determinar los efectos de un programa de formación en promoción de valores y políticas organizacionales a partir del derecho público en instituciones de seguridad ciudadana. 


\section{REFERENCIAL TEÓRICO}

\section{Valores}

En el horizonte de todo comportamiento humano se halla algo que da sentido a la actuación individual y social de las personas y explica el desarrollo cultural de las comunidades humanas; esto son precisamente los valores. Sin ellos la vida carecería de sentido, la convivencia sería imposible y el ser humano perdería completamente su norte y dirección. Por eso la crisis de valores comporta una crisis de conciencia, del sentido de la vida, y de la sociedad en su conjunto. Al respecto Sastre (1994), citado por Izquierdo C. (1998, p. 5), "estos (Valores) son ejes fundamentales por los que se orienta la vida humana y constituyen, a su vez, la clave del comportamiento de la persona".

El ser humano mantiene su armonía y su estructura personal gracias a los valores que se han ido construyendo en su vida de relación con los demás y que le vinculan al resto del cuerpo social. Podemos decir entonces que, los valores son importantes porque:

1. Son patrones que sirven para guiar la vida de los hombres.

2. Orientan la actividad humana en las situaciones concretas de la vida.

3. Mediatizan la percepción que el hombre se forma de los demás y de él mismo. Sirven de base para que el hombre juzgue su entorno.

4. Permite evaluar, resolver conflicto y tomar decisiones.

5. Permite mantener y asaltar la autoestima.

6. Son metas ideales que trascienden las situaciones.

7. Son patrones normativos y determinantes de actitudes de conductas.

8. El mundo de los valores constituye la puerta de entrada al mundo de la trascendencia.

9. El sentido trascendente de los valores fundamenta la fraternidad y solidaridad humana.

10. El valor siempre se refiere al ser humano pero el valor moral trasciende a su portador (Izquierdo, 1998). 
Prosiguiendo con lo expuesto, Izquierdo, C (1998, p. 10) explica que "los valores constituyen los fundamentos de las concepciones del mundo y de la vida". Para Hernando, M (1999) (Citado por López y Torres, 2008, p. 56) "los valores son relativos, dependen de la época, el lugar y el tipo de sociedad... Cada momento histórico tiene sus propios valores, los cuales a su vez están abocados al cambio según pasa el tiempo".

\section{Políticas organizacionales}

Las políticas organizacionales permiten a la empresa contar una visión que regule el comportamiento de sus miembros, en este sentido, Medina (2012), la define como:

La orientación o directriz que debe ser divulgada, entendida y acatada por todos los miembros de la organización, en ella se contemplan las normas y responsabilidades de cada área de la organización. Las políticas son guías para orientar la acción; son lineamientos generales a observar en la toma de decisiones, sobre algún problema que se repite una y otra vez dentro de una organización. En este sentido, las políticas son criterios generales de ejecución que complementan el logro de los objetivos y facilitan la implementación de las estrategias. Las políticas deben ser dictadas desde el nivel jerárquico más alto de la empresa ( $p$. 1).

Por consiguiente, se hace necesario que la empresa cuente con políticas organizacionales claras y establecidas para regular el marco de movilización interna de la organización, así mismo, Medina (2012), comenta que los beneficios de contar con políticas organizacionales, permite:

1. Aseguran un trato equitativo para todos los empleados.

2. Generan seguridad de comunicación interna en todos los niveles.

3. Es fuente de conocimiento inicial, rápido y claro, para ubicar en su puesto nuevos empleados.

4. Facilita una comunicación abierta y promueve la honestidad.

5. Desarrolla la autoridad, poder y liderazgo.

6. Asegura la confianza, transparencia, objetividad y aprendizaje.

7. Son indispensables para una adecuada delegación de autoridad.

8. Reflejan la imagen de la empresa y deben reajustarse a tiempo. 
Es así que por medio de las políticas organizacionales se puede fomentan valores con la finalidad de estructurar una empresa en capacidad de regular el comportamiento y clima organizacional como factores de convivencia para el establecimiento de acciones favorables que contribuyan al fomento de la productividad en un marco de referencia moral en cuanto a los servicios que presta a la comunidad. En complemento, Litwin, (1994), citado por Sandoval (2004) define el clima organizacional como la "cualidad o propiedad de ambiente organizacional que es percibido o experimentado por los miembros de la organización y que influyen en su comportamiento" (p.210).

Esto implica contar con talento humano en capacidad de convivir en valores y en proporcionalidad a las políticas organizacionales establecidas, con la finalidad de proyectar una imagen organizacional hacia el cliente externo (sociedad) que sea favorable según la filosofía de gestión de la institución. Goncalves (2002) describe:

El conocimiento del clima organizacional proporciona retroinformación acerca de los procesos que determinan los comportamientos organizacionales, permitiendo además, introducir cambios planificados tanto en las actitudes y conductas de los miembros, como en la estructura organizacional o en uno o más de los subsistemas que la componen" (p.47)

De lo expuesto, se basa en que el clima organizacional influye en el comportamiento palpable de los miembros, a través de percepciones estabilizadas que se introduce en el entorno de la organización lo que acondicionan la realidad y los niveles de motivación laboral y rendimiento profesional. Por ende, el clima organizacional se refiere al conjunto de propiedades medibles de un ambiente de trabajo, según son percibidas por quienes trabajan en él. Para la institución, es importante medir y conocer el clima organizacional, por cuanto este puede impactar significativamente los resultados.

\section{METODOLOGÍA DE LA INVESTIGACIÓN}

El tipo de investigación obedece al explicativo, Hernández, Fernández, y Baptista (2014), lo describen:

Los estudios explicativos van más allá de la descripción de conceptos o fenómenos o del establecimiento de relaciones entre conceptos; es 
decir, están dirigidos a responder por las causas de los eventos y fenómenos físicos o sociales. Como su nombre lo indica, su interés se centra en explicar por qué ocurre un fenómeno y en qué condiciones se manifiesta o por qué se relacionan dos o más variables (95).

En tal sentido la investigación procura comprobar o negar la hipótesis general, la cual se concibe desde los efectos de un programa para la promoción de valores y políticas organizacionales a partir del derecho público en instituciones de seguridad ciudadana, siendo posible explicar a partir de los resultados obtenidos el comportamiento de la variable de estudio.

El diseño utilizado en la investigación fue el cuasi experimental de pre prueba, postprueba y grupo control, definido por Hernández, Fernández y Baptista (2008) del siguiente modo:

Los sujetos son asignados al azar a los grupos, después a éstos se les administra simultáneamente la pre prueba, un grupo recibe el tratamiento experimental y otro no (es el grupo de control); y finalmente se les administra, también simultáneamente una postprueba (p. 193).

En este sentido, se trabajó con dos grupos de estudiantes, a uno se le aplicó el tratamiento $(\mathrm{x})$ y a otro no se le aplicó el tratamiento, sin embargo, a ambos grupos se les aplicó una pre prueba (antes de aplicar el tratamiento) y una post prueba (una vez aplicado el tratamiento).

Esto con la finalidad de tener un patrón de comparación y poder así, comparar los resultados obtenidos con otro grupo, de ese modo, se estableció una relación estadística que permitió determinar la diferencia obtenida en los momentos que se aplicó la prueba y obtener datos que permitieron explicar qué tan significativo o no, resultó la aplicación del tratamiento (programa). En concordancia con lo realizado, Hernández, Fernández y Baptista (2008) explican que:

Las puntuaciones de las pre pruebas pueden usarse para fines de control en el experimento, al compararse las pre pruebas de los grupos se puede evaluar qué tan adecuada fue la aleatorización. La segunda ventaja reside en que se puede analizar el puntaje ganancia de cada grupo (la diferencia entre la pre prueba y la postprueba) (p. 193).

Siguiendo este orden de ideas, se realizó una comparación estadística donde se estableció las diferencias entre los grupos de tratamiento y control. En 
consecuencia, el diagrama de este diseño, de acuerdo a Hernández, Fernández y Baptista (2008, p. 193) se estableció de la siguiente manera:

- G1 = Grupo al cual se le aplicó el tratamiento

- 01 = Momento de la pre prueba del RG1

- $\quad \mathrm{X}=$ Tratamiento aplicado a RG1

- 02 = Momento de la post prueba a RG1

- G2 = Grupo al cual no se le aplicó el tratamiento

- 03 = Momento de la pre prueba del RG2

- $\quad$ - = Tratamiento no aplicado a RG2

- 04 = Momento de la post prueba a RG2

La población es definida por Hernández, Fernández y Baptista (2008), como un "conjunto de casos que concuerdan con determinadas especificaciones" (239), siendo una población focalizada de 70 participantes, pertenecientes a instituciones de seguridad ciudadana de Manta, Ecuador, divididos en grupos de 35 personas para el establecer los grupos G1 y G2, para lo cual se utilizó el azar simple como muestreo aleatorio, por lo cual, todos los participantes tuvieron la misma posibilidad de estar en uno de los dos grupos.

\section{RESULTADOS}

Se aplicó una prueba de hipótesis utilizando un nivel de significancia de 5\%. Las hipótesis planteadas son:

Nula H0: No existe diferencias significantes entre ambos grupos, lo que es igual no funcionó el tratamiento.

Alterna H1: Existen diferencias significantes entre ambos grupos, lo que es igual, sí funcionó el tratamiento.

La prueba utilizada fue la $T$ de Student, la cual fue calculada en el paquete estadístico SPSS 25.0

T de Student grupo control y experimental

Cuadro 1 
Prueba de

Levene para

la igualdad de

varianzas

Prueba T para la igualdad de medias

La

tabl

95\% Intervalo de

a

confianza para la

diferencia

Sig. Diferencia Error típ. de la

F Sig

\begin{tabular}{|c|c|c|c|c|c|c|c|c|}
\hline \multirow{2}{*}{14,861} & \multirow[t]{2}{*}{,000 } &,- 430 & 55 & ,669 &,- 32010 & 74359 & $-1,81029$ & 1,17009 \\
\hline & & \multicolumn{2}{|c|}{$-, 45346,466$} & ,652 &,- 32010 & ,70611 & $-1,74104$ & 1,10084 \\
\hline \multirow[t]{2}{*}{5,095} & ,028 & 53,974 & 55 &, 000 & 27,54218 & ,51028 & 26,51955 & 28,56482 \\
\hline & & 55,324 & 54,422 &, 000 & 27,54218 & 49784 & 26,54426 & 28,54011 \\
\hline
\end{tabular}

iguales. En dichos resultados el valor de significancia bilateral fue de 0,669 el cual es $>0,05$, lo que indica que no existen diferencias significativas entre ambos grupos (experimental y control) para el momento del pre test.

En las filas de la tabla de "pruebas independientes" concerniente al pos test, el valor de Levene también fue de $<0,05$, por lo tanto, se asumen varianzas iguales. En este caso, el nivel de significancia bilateral cambió a "0", lo que es lo mismo $<0,05$, lo que indica diferencias significativas en ambos grupos (experimental y control).

Se rechaza $\mathrm{HO}$ y se acepta $\mathrm{H} 1$, lo que indica que el tratamiento aplicado resultó favorable a la muestra objeto de estudio.

\section{DISCUSIÓN}

Al existir significancias en los grupos a la luz de la aplicación del tratamiento, se evidencia que los participantes se encuentran en capacidad cognitiva de asumir un rol de seguridad ciudadana desde una concepción basada en valores para la vida, así como conocer la importancia de las políticas organizacionales de la institución, basadas desde el derecho público, con lo cual cuentan con las herramientas para desempeñar una función ética dentro y fuera de la institución, concordando con la posición de García, García \& Isea (2018), al indicar la necesidad de un liderazgo 
organizacional basado en una nueva ética que permita ejercer funciones en pertinencia a los derechos humanos, propiciándose la dignidad humana como factor para el crecimiento humano integral, tal como proponen Aldana Zavala\& Isea (2018).

\section{CONCLUSIONES}

La política organizacional constituye uno de los factores determinantes de los procesos organizativos, de gestión, cambio e innovación. Adquiere relevancia por su repercusión inmediata, tanto en los procesos como en los resultados, lo cual incide directamente en la calidad del propio sistema y su desarrollo.

Los esfuerzos para mejorar la vida laboral constituyen tareas sistemáticas que llevan cabo las organizaciones para proporcionar a los empleados una oportunidad de mejorar sus puestos y contribución a la empresa, es un ambiente de mayor confianza y respeto, es por ello que el clima organizacional se refiere al ambiente propio de la organización.

Desde el derecho público se promueve servidores públicos de seguridad ciudadana, en consonancia con valores y políticas organizacionales que les permitan abordar a las personas dentro y fuera de la institución en un marco de cordialidad, respeto, como valores fundamentales para establecer un principio integrador de los derechos humanos como factor elemental para el desenvolvimiento de las funciones públicas de seguridad.

En resumen, se tiene que los efectos del programa de formación en promoción de valores y políticas organizacionales a partir del derecho público en instituciones de seguridad ciudadana, fueron efectivos a partir de la prueba de comparativa aplicada, con lo cual se considera que la ciudadanía cuenta con servidores en posibilidad de abordar desde el derecho público, una asertiva relación interpersonal basada en valores para el buen vivir.

\section{REFERENCIAS CONSULTADAS}

1. Aldana Zavala, J., \& Isea, J. (2018). Derechos Humanos y Dignidad Humana. IUSTITIA SOCIALIS, 3(4), 8-23. Recuperado de http://fundacionkoinonia.com.ve/ojs/index.php/lustitia Socialis/article/view/119 $\underline{101}$ 
2. Contreras, E. (2005) Principios y Valores del proceso Bolivariano: Agenda para una discusión pendiente. Valencia. Editorial Vadell Hermanos Editores C.A.

3. García., L., García, J., \& Isea, J. (2018). La función ética y social del gerente educativo. Revista Arbitrada Interdisciplinaria Koinonía, 2(3), 80-91. Recuperado de http://fundacionkoinonia.com.ve/ojs/index.php/revistakoinonia/article/view/53/4 $\underline{0}$

4. Hernando, M. (1999) Estrategias para educar en valores. Madrid. Editorial CCS.

5. Hernández, R., Fernández, C., Baptista, P. (2014). Metodología de la Investigación. México: Editorial Mc - Graw - Hill Interamericana. Sexta edición.

6. Izquierdo, C. (1998) El mundo de los valores. Caracas- Venezuela. Editorial Paulinas Distribuidoras.

7. Medina Mariana. (2012). Política organizacional. Concepto y esquema en la empresa. Recuperado de https://www.gestiopolis.com/politica-organizacionalconcepto-y-esquema-en-la-empresa/

\section{REFERENCES CONSULTED}

1. Aldana Zavala, J., \& Isea, J. (2018). Human Rights and Human Dignity. IUSTITIA SOCIALIS, 3 (4), 8-23. Retrieved from http://fundacionkoinonia.com.ve/ojs/index.php/lustitia Socialis/article/view/119 $\underline{101}$

2. Contreras, E. (2005) Principles and Values of the Bolivarian process: Agenda for a pending discussion. Valencia. Editorial Vadell Hermanos Editores C.A.

3. García., L., García, J., \& Isea, J. (2018). The ethical and social function of the educational manager. Interdisciplinary Arbitrated Review Koinonía, 2 (3), 8091. Recovered from http://fundacionkoinonia.com.ve/ojs/index.php/revistakoinonia/article/view/53/4 $\underline{0}$

4. Hernando, M. (1999) Strategies to educate in values. Madrid. CCS editorial.

5. Hernández, R., Fernández, C., Baptista, P. (2014). Investigation methodology. Mexico: Editorial Mc - Graw - Interamerican Hill. Sixth edition. 
6. Izquierdo, C. (1998) The world of values. Caracas Venezuela. Editorial Paulinas Distribuidoras.

7. Medina Mariana. (2012). Organizational Policy Concept and scheme in the company. Recovered from https://www.gestiopolis.com/politicaorganizacional-concepto-y-esquema-en-la-empresa/

(C2019 por los autores. Este artículo es de acceso abierto y distribuido según los términos y condiciones de la licencia Creative Commons Atribución-NoComercial-Compartirlgual 4.0 Internacional (CC BY-NC-SA 4.0) (https://creativecommons.org/licenses/by-nc-sa/4.0/). 